\title{
Large Band Edge Tunability in Colloidal Nanoplatelets
}

Qunfei Zhou, ${ }^{\dagger \ddagger}$ Yeongsu Cho, " Shenyuan Yang, ${ }^{\S} \|$ Emily A. Weiss, ${ }^{\perp}$

Timothy C. Berkelbach, ${ }^{*, \#, @ ~ a n d ~ P i e r r e ~ D a r a n c e t *, ~}{ }^{*}, \triangle$

$\dagger$ Materials Research Science and Engineering Center, Northwestern University, Evanston, IL 60208, USA

$\ddagger$ Center for Nanoscale Materials, Argonne National Laboratory, Argonne, IL 60439, USA

【 Department of Chemistry and James Franck Institute, University of Chicago, Chicago, IL 60637, USA

$\S$ State Key Laboratory of Superlattices and Microstructures, Institute of Semiconductors, Chinese Academy of Sciences, Beijing 100083, China

\|Center for Materials Science and Optoelectronics Engineering, University of Chinese Academy of Science, Beijing 100049, China

$\perp$ Department of Chemistry and Department of Materials Science and Engineering, Northwestern University, Evanston, IL 60208, USA \#Department of Chemistry, Columbia University, New York, NY 10027, USA

@ Center for Computational Quantum Physics, Flatiron Institute, New York, NY 10010, $U S A$

$\triangle$ Northwestern Argonne Institute for Science and Engineering, Evanston, IL 60208, USA

E-mail: tim.berkelbach@gmail.com; pdarancet@anl.gov 


\section{Contents}

$\begin{array}{lr}\text { Further computational Details } & 3\end{array}$

$\begin{array}{ll}\text { Ligand Binding Energies } & 4\end{array}$

Band Edge Shifts and Band Gaps

$\begin{array}{lr}\text { Charge Densities } & 8\end{array}$

DOS and pDOS for CdSe NPLs-MoS ${ }_{2}$ van der Waals Heterojunctions $\quad 9$

$\begin{array}{ll}\text { Theoretical estimation of the band gap and optical gap } & 10\end{array}$

$\begin{array}{ll}\text { Bibliography } & 12\end{array}$ 


\section{Further Computational Details}

The pseudopotentials used in this work are Optimized Norm-Conserving Vanderbilt Pseudopotentials (ONCVP) ${ }^{1}$ obtained from: http://www.quantum-simulation.org/potentials/sg15_oncv/. The valence electrons for each elements are: $\mathrm{Cd} 4 s^{2} 4 p^{6} 4 d^{10} 5 s^{2}$, Se $4 s^{2} 4 p^{4}, \mathrm{O} 2 s^{2} 2 p^{4}$, S $3 s^{2} 3 p^{4}$, $\mathrm{N} 2 s^{2} 2 p^{3}$, and $\mathrm{F} 2 s^{2} 2 p^{5}$. Convergence test results on k-points and plane wave cutoff energy (ecutwfc) are shown in Fig. S1.

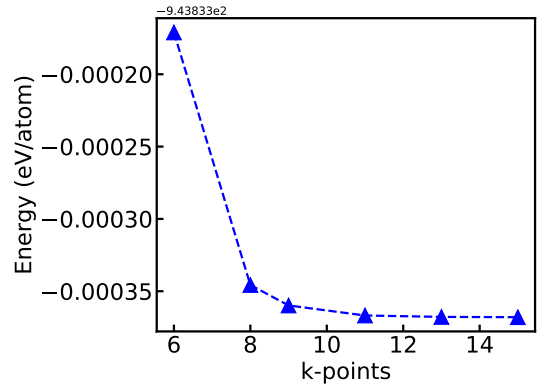

(a)

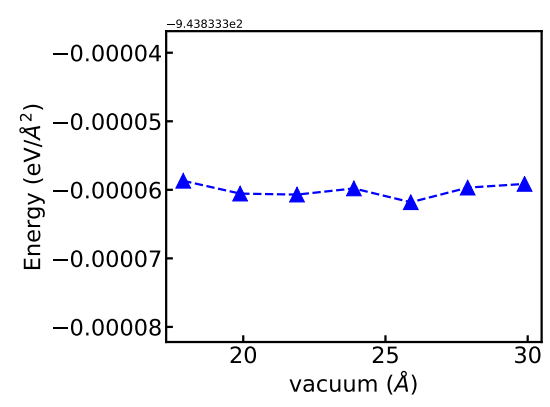

(d)

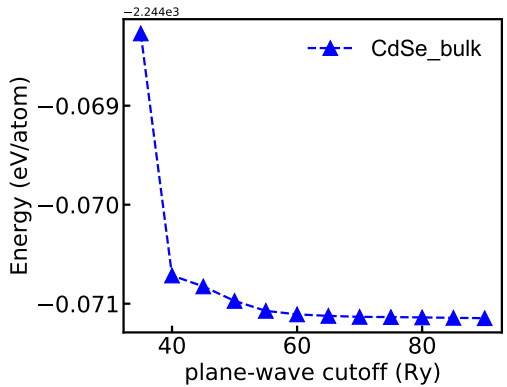

(b)

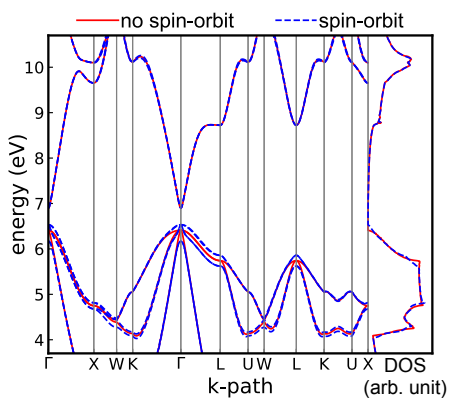

(e)

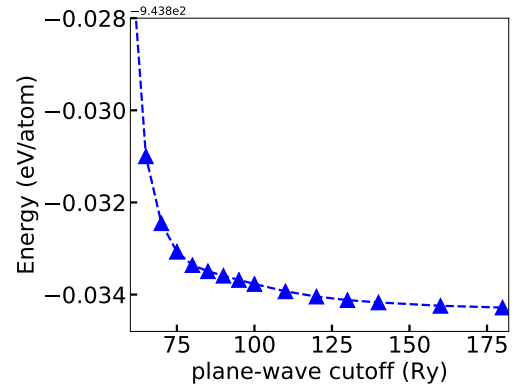

(c)

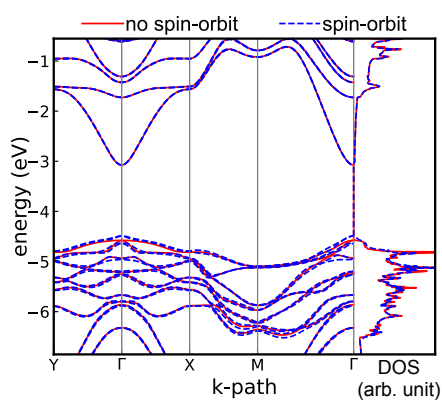

(f)

Figure S1: (a) Convergence of total energy with k-points. Total energy as a function of ecutwfc for (b) bulk zinc blende CdSe, and (c) CdSe NPLs with ligands. (d) Convergence of total energy with length of vacuum region. Band structures and density of States (DOS) with and without considering spin-orbit coupling for (e) bulk zinc blende CdSe, and (f) CdSe NPLs with $\mathrm{MeS}^{-}$ligands. The spin-orbit coupling effects shift the valence band up by about $0.1 \mathrm{eV}$, for both CdSe bulk and NPLs. But no other notable differences are observed otherwise. 


\section{Ligand Binding Energies}

The binding energy is calculated as follows:

$$
E_{b}=\left\{E[\text { ligand } / C d S e(001)]-E[C d S e(001)]-n E[\text { ligand }]+(n / 2) E\left[H_{2}\right]\right\} / n
$$

where $n$ is the number of ligands at the surface, and $E[C d S e(001)]$ is the total energy of non-passivated surface.

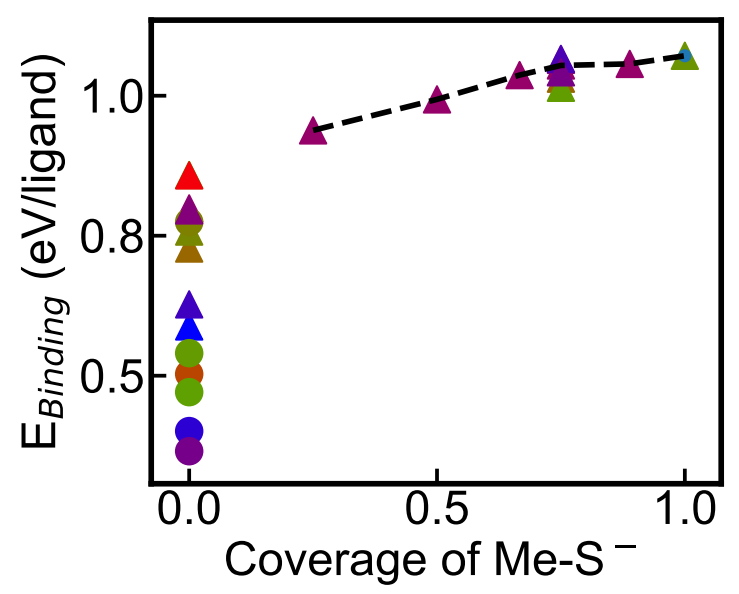

Figure S2: Binding energies of different types of ligands at CdSe NPLs surface calculated according to Eq 1. The colors of each points correlates with that in Fig. 2 according to their intrinsic dipole moments. Triangles and filled circles are for NPLs with R-S ${ }^{-}$and R-Ac ${ }^{-}$ ligands, respectively. The binding energy is largest for CdSe NPLs with Me-S- ligand, and decreases with decreasing coverage of $\mathrm{Me}^{-} \mathrm{S}^{-}$, and followed by that with mixed ligands of different coverages. Black dashed line is included as guides for the eye. 


\section{Band Edge Shifts and Band Gaps}

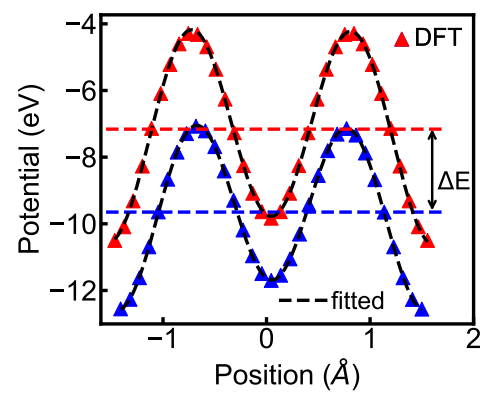

(a)

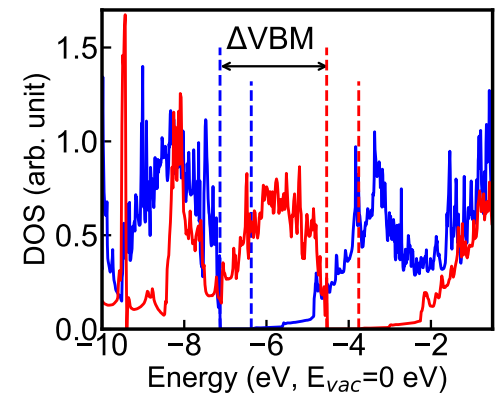

(b)

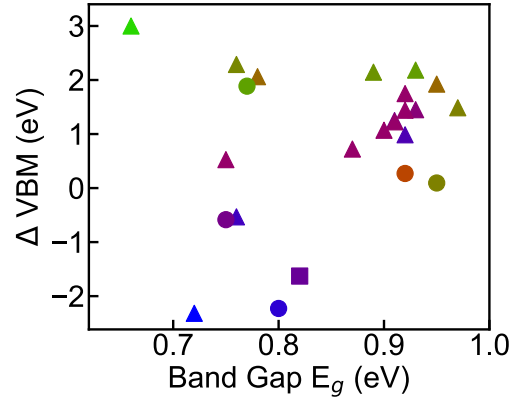

(c)

Figure S3: (a) Planar-averaged electrostatic potential, same as that in Figure 1 (c), but only include that for the one unit at the center of CdSe NPLs. The position of electrostatic potential for each NPLs is determined by fitting the DFT-calculated data (red triangles) for this one unit at the center with sin-wave functions (black dashed lines), and taking the average value from the fitting curve. Accurate position of electrostatic potential is predicted in this way even if there are no DFT-caluclated values at the peak/valley positions. The potential energies are all referenced to vacuum level. (b) Density of states (DOS) for CdSe NPLs passivated with $\mathrm{C}_{6} \mathrm{H}_{5}-\mathrm{S}^{-}$(red) and $\mathrm{CF}_{3} \mathrm{C}_{6} \mathrm{H}_{4}-\mathrm{S}^{-}$(blue) ligands, same as that in Figure 1 (b) but with superimposed DOS. The vacuum potential is set as zero. (c) The valence band edge shift $\triangle$ VBM and band gap for 6.5 ML CdSe NPLs with 20 ligands studied in this work. This shows independence of the band edge shift with band gap. The color of the data points are correlated with that of the ligands as shown in Figure 2 (a).

Table S1: In-plane and out-of-plane lattice constant, a and c, respectively, and lattice strain, $\Delta \epsilon_{x, y}$ and $\Delta \epsilon_{z}$, respectively, along with bandgap variations for NPLs of thickness 3.5 ML and 4.5 ML. Results are comparing with experiments ${ }^{2}$ for CdSe NPLs with phosphonic acid (PA) and oleic acid (OA) ligands. Ligands in this work are counterparts of that in experiments, only substituting the long-chain functional group by $\mathrm{H}$ keeping the binding groups the same. $\Delta \epsilon=(a[P A]-a[O A]) / a[O A] \times 100 \%$.

\begin{tabular}{|c|c|c|c|c|c|c|c|c|c|}
\hline $\mathrm{n}(\mathrm{L})$ & ligand & $\begin{array}{c}\text { Exp. }^{2} \\
\mathrm{a}(\AA) \mathrm{c}(\AA)\end{array}$ & $\begin{array}{l}\text { this work } \\
\text { a }(\AA) \text { c }(\AA)\end{array}$ & $\Delta \epsilon_{x, y}$ & $\epsilon_{z}, \%$ & $\begin{array}{c}\text { this } \mathrm{w} \\
\%\end{array}$ & $\begin{array}{l}\Delta \epsilon_{x, y}, \\
\%\end{array}$ & $\begin{array}{c}\text { Exp. }^{2} \\
E_{g}(\mathrm{eV})\end{array}$ & $\begin{array}{l}\text { this work } \\
E_{g}(\mathrm{eV})\end{array}$ \\
\hline 3.5 & $\mathrm{OA}$ & 6.176 .02 & 6.3215 .963 & 0 & 0 & 0 & 0 & 2.75 & 2.781 \\
\hline 3.5 & $\mathrm{PA}$ & $5.99 \quad 6.27$ & $6.276 \quad 6.146$ & -2.89 & 4.3 & -0.720 & 3.073 & 2.48 & 2.589 \\
\hline 4.5 & $\mathrm{OA}$ & $6.19 \quad 5.94$ & 6.3035 .998 & 0 & 0 & 0 & 0 & 2.46 & 2.498 \\
\hline 4.5 & PA & $6.02 \quad 6.21$ & $6.218 \quad 6.244$ & -2.79 & 4.47 & -1.344 & 4.098 & 2.32 & 2.314 \\
\hline
\end{tabular}

The band gap variations with ligands can be attributed to the effect of strain and ligandNPLs coupling. As shown in Fig. S5 (b), the lattice constant (LC) of CdSe NPLs with MeS ${ }^{-}$ 

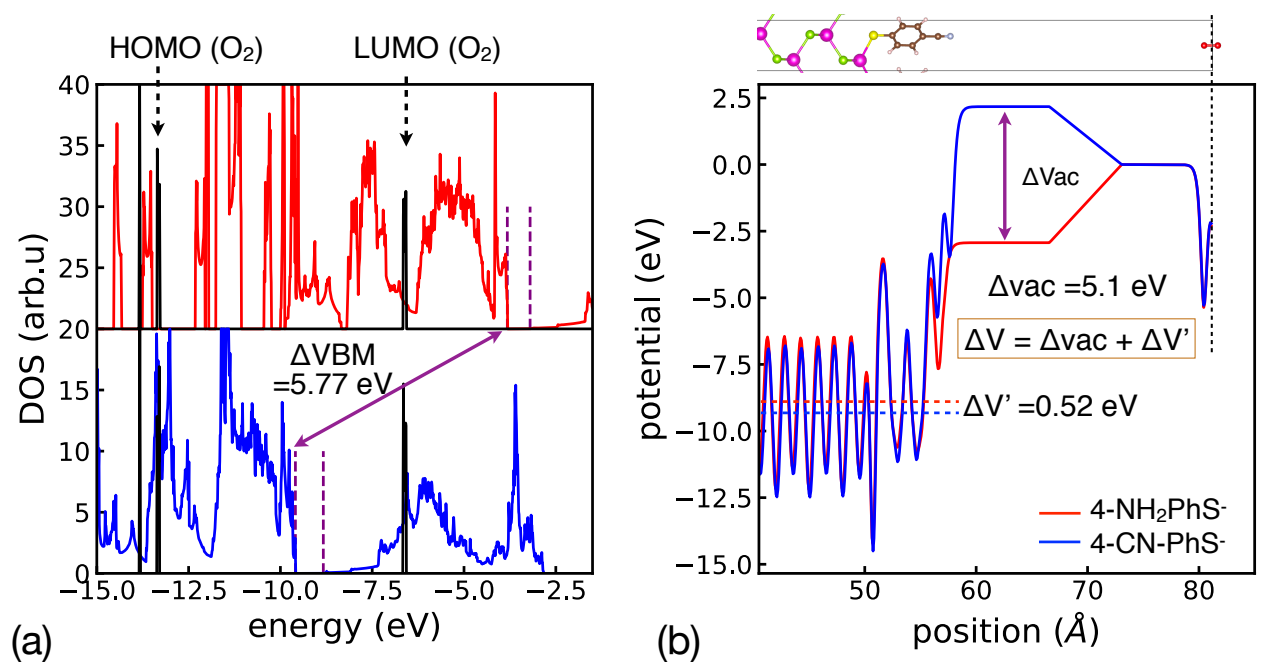

Figure S4: (a) Density of States (DOS) and (b) potential energies for 6.5 ML CdSe NPL with $4-\mathrm{NH}_{2}-\mathrm{PhS}^{-}$(red) and 4-CN-PhSS${ }^{-}$(blue) ligands, respectively, both includes $\mathrm{O} 2$ molecule in the vacuum region far away (about $21 \AA$ ) from the NPL surface, as shown at the top of (b). Black DOS are for O2. Energy in (a) are referenced to their vacuum levels. And in (b), the potentials of $\mathrm{O} 2$ are aligned for the two structures. As shown in (a), the shift in band edges are $\Delta \mathrm{VBM}=5.77 \mathrm{eV}, \Delta \mathrm{CBM}=5.63 \mathrm{eV}$. And the potential energy shift for the two NPLs is $5.62 \mathrm{eV}$, consistent with the band edge shifts.

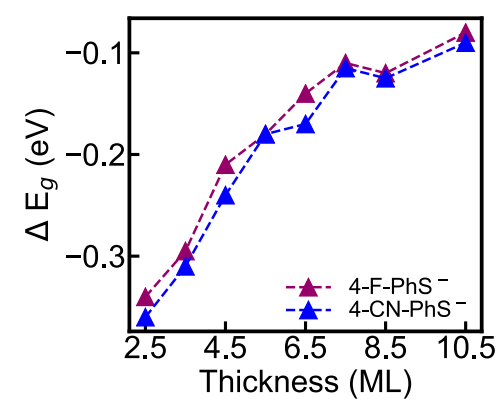

(a)

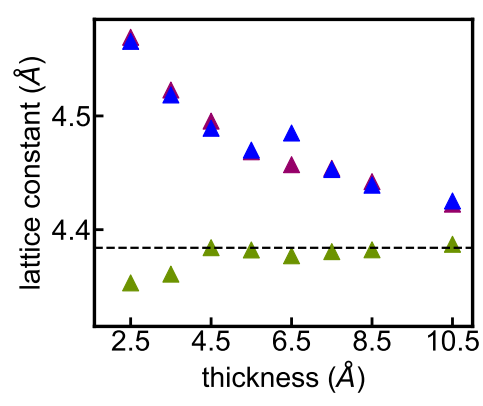

(b)

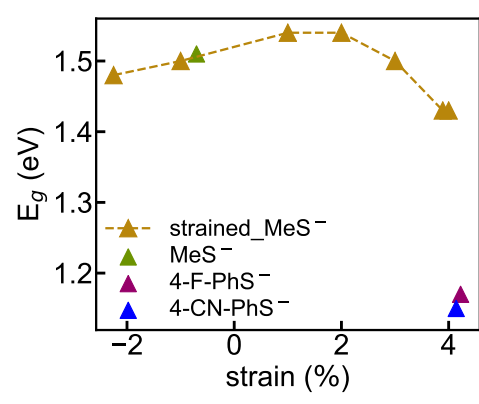

(c)

Figure S5: (a) Bandgap difference $\Delta E_{g}$ between CdSe NPLs with 4-F-PhS- (purple), 4-CN$\mathrm{PhS}^{-}$(blue) ligands and $\mathrm{MeS}^{-}$ligand. (b) Lattice constants of CdSe NPLs with ligands of $\mathrm{MeS}^{-}$(green), 4-F-PhS ${ }^{-}$(purple), and 4-CN-PhS- (blue), and their changes with thickness. CdSe NPLs with $\mathrm{MeS}^{-}$ligand have lattice constants very close to that of the bulk (black dashed line). (c) The influence of the change of lattice constant, or strain, which is calculated as: $(\mathrm{LC}(\mathrm{NPLs})-\mathrm{LC}($ bulk $)) / \mathrm{LC}($ bulk $) \times 100 \%$.

ligands are very close to that for bulk CdSe for thicknesses from 4.5 ML to 10.5 ML, though small reduction of LC for 2.5-3.5 ML. However, for CdSe NPLs with 4-F-PhS ${ }^{-}$ligands, LC 

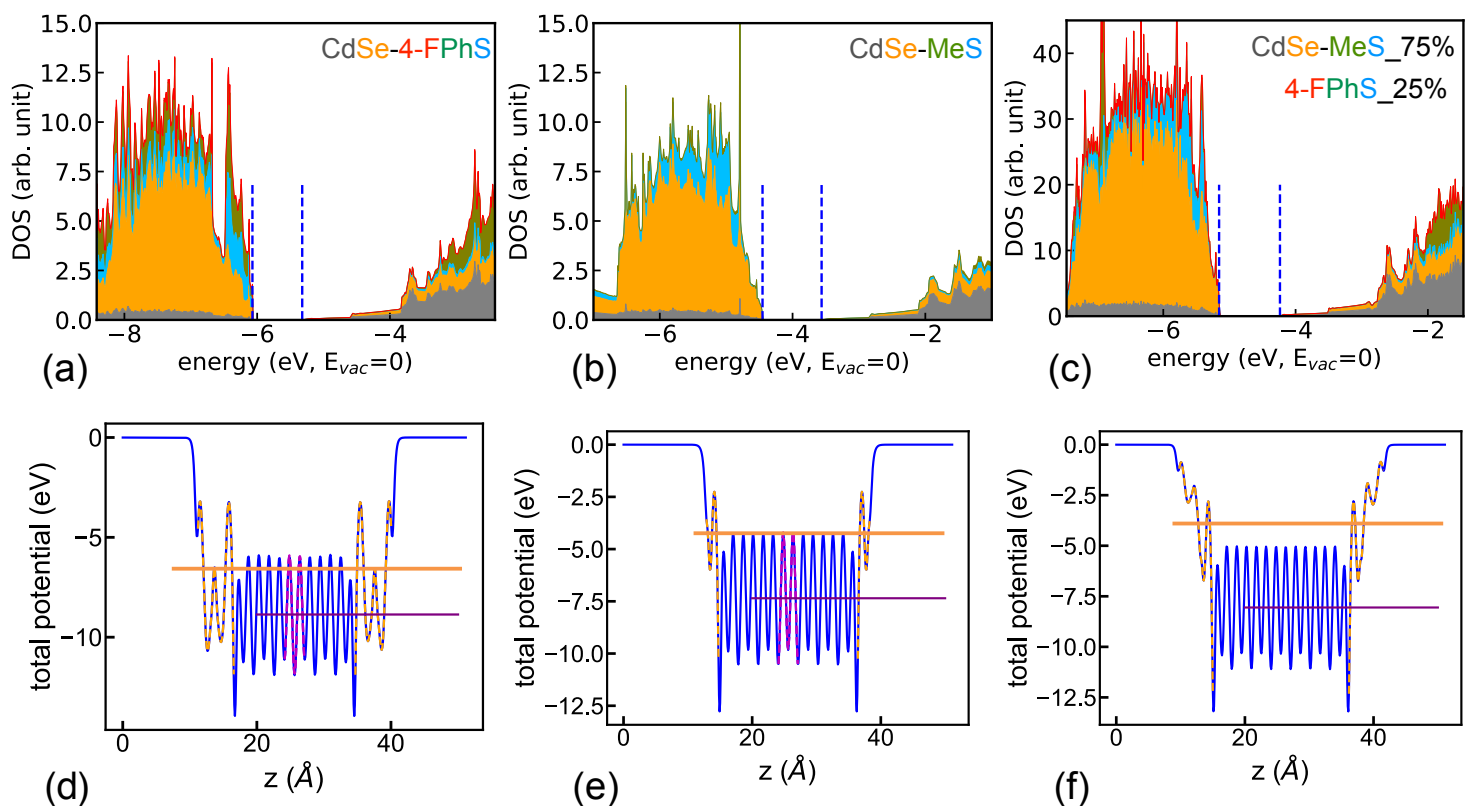

(e)

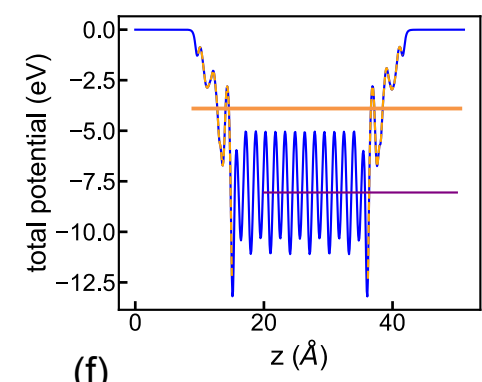

Figure S6: Projected-density of states (pDOS) for CdSe NPLs with ligands of (a) 4-F-PhS ${ }^{-}$, (b) $\mathrm{MeS}^{-}$, and (c) $25 \% \mathrm{MeS}^{-}$and $75 \%$ 4-F-PhS ${ }^{-}$, and their corresponding Hartree potentials (c)-(e), respectively. The band gaps for CdSe NPLs from (a) to (c) are $0.75 \mathrm{eV}, 0.89 \mathrm{eV}$, and $0.92 \mathrm{eV}$, respectively. Contributions of different elements are shown with different colors: Cd (gray), Se (orange), S (skyblue), benzene or -CH3 (olive), F (red). The potentials for CdSe center and ligands are marked in dashed purple and orange lines, respectively.

increases with decrease of thickness and are all larger than that of bulk, indcating strong repulsion among the ligands creating large strain for NPLs. And this effect enhances with decrease in thickness, resulting in larger differences in band gaps with thinner NPLs as shown in Fig. S5(a). The effect of strain is directly calculated for 2.5 ML NPLs with MeS ${ }^{-}$ligand as shown in Fig. S5(c). The band gap of NPLs with $\mathrm{MeS}^{-}$, though having same strain as that with $4-\mathrm{F}_{-} \mathrm{PhS}^{-}$, is much larger, which is attributed to the ligand-NPL coupling as shown in Fig. S6.

In order to better compare with reported experimental data on ligand-induced optical bandgap shifts, we compared the in-plane and out-of-plane lattice parameters and bandgaps for CdSe NPLs with phosphonic acid and oleic acid, see Table S1. As shown in Table S1, the optical bandgaps from this work are in good agreement with experiments. Meanwhile, comparing with oleic acid ligands, the phosphonic acid ligands induced in-plane compression 
and out-of-plane expansion, leading to smaller quantum confinement effect, therefore smaller bandgaps.

Large contribution of the ligand orbitals - from both S 3p orbitals and the phenyl ring $(\mathrm{Ph})$ - to the highest occupied states are observed for CdSe with 4-F-PhS ${ }^{-}$ligand, Fig. S6(a). While Me-S ${ }^{-}$and mixed $\left(x \mathrm{MeS}^{-}+y 4-\mathrm{F}-\mathrm{PhS}^{-}\right)$ligands have minimal contributions to the highest occupied states, Fig. S6 (b)-(c). This indicates strong coupling between the CdSe NPLs and 4-F-PhS ligands, especially with ligand orbitals that have significant $\pi$ character, which is consistent with that for QDs, ${ }^{3-5}$ and leading to smaller band gap than that with $\mathrm{MeS}^{-}$and mixed ligands. 


\section{Charge Densities}

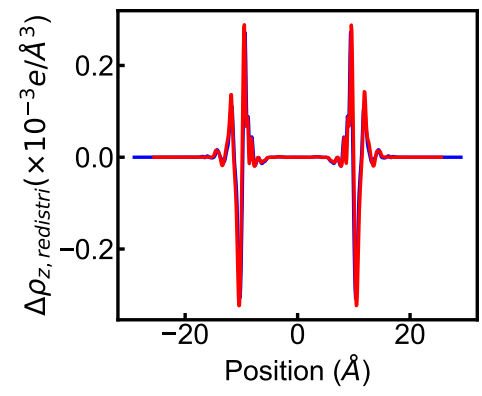

(a)

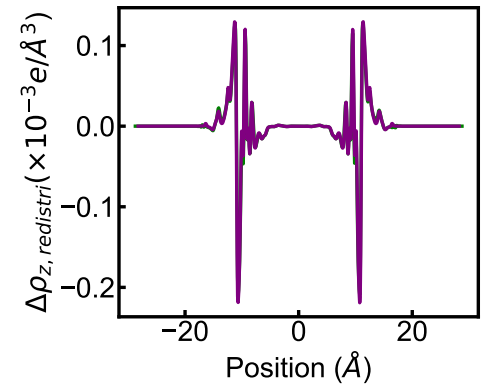

(b)

Figure S7: (a) Redistribution of charge density $\left(\Delta \rho_{z, \text { redistri }}\right)$ induced by the chemical interaction between the ligand and CdSe. $\Delta \rho_{z \text {,redistri }}$ here is plotted in the same way as that in Figure 2 (c), but both the charge density of CdSe and respective ligands are subtracted from that of the total charge density of the passivated CdSe. Red and Blue curves are for CdSe NPLs with 4-F-PhS ${ }^{-}$and $4-\mathrm{CF}_{3}-\mathrm{PhS}^{-}$, respectively. (b) same as in (a) but for CdSe NPLs passivated with $4-\mathrm{F}-\mathrm{PhAc}^{-}$(purple) and $4-\mathrm{CF}_{3}-\mathrm{PhAc}^{-}$(green). As a result, charge redistribution depends only on the binding group: (a) thiol and (b) carboxylate groups, but not on dipoles or other functional groups. 


\section{DOS and pDOS for CdSe NPLs-MoS 2 van der Waals}

\section{Heterojunctions}

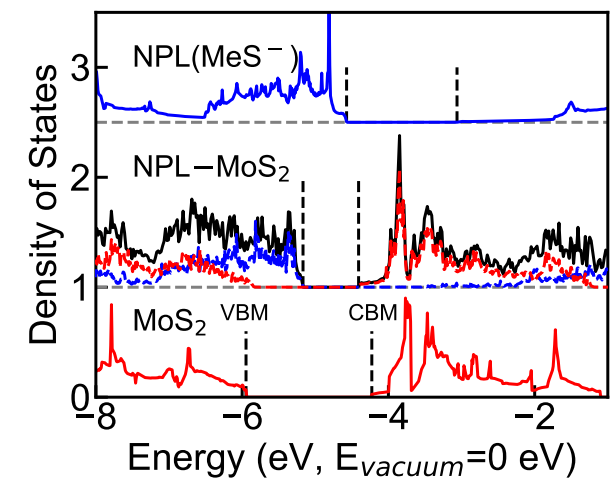

(a)

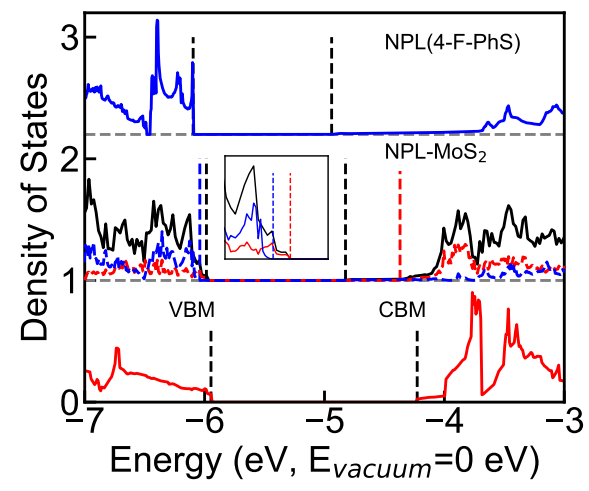

(b)

Figure S8: (a) Density of states (DOS) for individual MeS- ${ }^{-}$passivated 2ML CdSe NPL (top), individual 1ML $\mathrm{MoS}_{2}$ (bottom), and heterostructure consisting of the two. The projected DOS of NPLs and $\mathrm{MoS}_{2}$ in the heterostructures are shown in dashed blue and red lines, respectively. Same are shown in (b) for $2 \mathrm{ML}$ CdSe NPL (top) passivated with 4-F-PhS ${ }^{-}$. Black vertical dashed lines depict VBM and CBM. Both heterojunctions are type II band alignments, same as expected from their intrinsic band edges as shown in Fig. 3 (b) 


\section{Theoretical estimation of the band gap and optical gap}

The procedure to calculate thickness-dependent and environmentally-sensitive self-energy corrections and exciton binding energies follows our previous work. ${ }^{6}$ A CdSe NPL is approximated as an infinite CdSe slab sandwiched by ligand layers and immersed in a solvent, each with a uniform dielectric constant, as depicted in Fig. S9. The screened Coulomb interaction $W_{N P L}\left(\rho ; z, z^{\prime}\right)$ of the corresponding layered system is exactly solved by the electrostatic transfer matrix method, ${ }^{7}$ where $\rho$ is the in-plane separation and $z$ and $z^{\prime}$ are the out-of-plane positions of the two charges. For simplicity, we assumed both charges to be located at the center of the NPL, i.e. $z=z^{\prime}=0$.

The valence and conduction band edges are given by

$$
E_{\mathrm{VBM}}=E_{\mathrm{VBM}}^{\mathrm{DFT}}-\Sigma_{\text {bulk }}-\delta \Sigma \quad E_{\mathrm{CBM}}=E_{\mathrm{CBM}}^{\mathrm{DFT}}+\Sigma_{\text {bulk }}+\delta \Sigma
$$

where $\Sigma_{\text {bulk }}$ is a self-energy shift that corrects the DFT bulk band gap of CdSe and

$$
\delta \Sigma=\frac{1}{2} \lim _{\rho \rightarrow 0}\left[W_{N P L}(\rho)-\frac{1}{\varepsilon_{\mathrm{CdSe}} \rho}\right]
$$

is a self-energy shift that accounts for the dielectric constrast between a CdSe slab and its

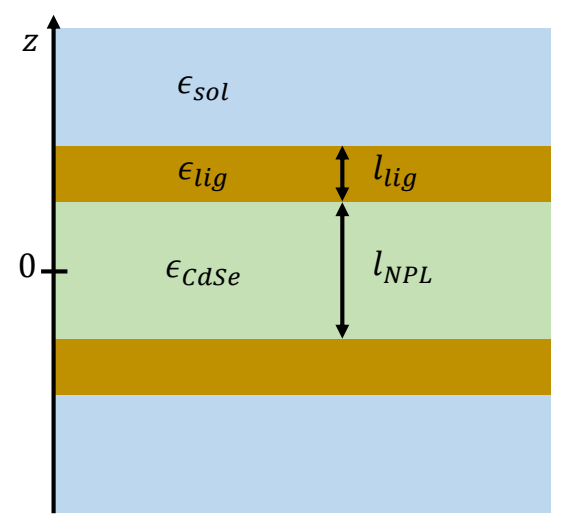

Figure S9: Electrostatic model of a CdSe NPL surrounded by ligands and immersed in solvent. 
environment. The expressions above assume that DFT correctly accounts for the band edge changes originating from carrier confinement and from the ligand dipoles, as discussed in the main text. In our calculations we use the following parameters ${ }^{8,9}$

$$
\begin{aligned}
2 \Sigma_{\text {bulk }} & =1.27 \mathrm{eV} \\
\varepsilon_{\mathrm{CdSe}} & =6.2 \\
\varepsilon_{\text {lig }} & =2.1 \\
\varepsilon_{\text {sol }} & =1,
\end{aligned}
$$

and the widths $l_{\mathrm{NPL}}$ and $l_{\text {lig }}$ are determined from the DFT-optimized structures of each slab with each ligand.

In order to calculate the optical gap for comparison with experimental measurements, we subtract the exciton binding energy from the quasiparticle band gap determined above. The exciton binding energy is calculated with a 2D effective mass Hamiltonian

$$
H=-\frac{1}{2 \mu} \nabla_{\rho}^{2}-W_{N P L}(\rho)
$$

where $\mu$ is the reduced mass of the exciton $\mu=m_{e} m_{h} /\left(m_{e}+m_{h}\right)$ and the effective mass of the electron and hole for CdSe are 0.12 and 0.18 respectively. ${ }^{10}$ With this Hamiltonian, the time-independent Schrodinger equation is solved on a radial spatial grid to determine the exciton binding energy. 


\section{Bibliography}

(1) Hamann, D. Phys. Rev. B 2013, 88, 085117.

(2) Antanovich, A.; Achtstein, A.; Matsukovich, A.; Prudnikau, A.; Bhaskar, P.; Gurin, V.; Molinari, M.; Artemyev, M. Nanoscale 2017, 9, 18042-18053.

(3) Giansante, C. J. Phys. Chem. C 2018, 122, 18110-18116.

(4) Giansante, C.; Infante, I.; Fabiano, E.; Grisorio, R.; Suranna, G. P.; Gigli, G. J. Am. Chem. Soc. 2015, 137, 1875-1886.

(5) Azpiroz, J. M.; De Angelis, F. ACS Appl. Mater. Interfaces 2015, 7, 19736-19745.

(6) Cho, Y.; Berkelbach, T. C. Phys. Rev. B 2018, 97, 1-6.

(7) Cavalcante, L.; Chaves, A.; Van Duppen, B.; Peeters, F.; Reichman, D. Phys. Rev. B 2018, 97, 125427.

(8) Even, J.; Pedesseau, L.; Kepenekian, M. Phys. Chem. Chem. Phys. 2014, 16, 2518225190.

(9) Muljarov, E.; Tikhodeev, S.; Gippius, N.; Ishihara, T. Phys. Rev. B 1995, 51, 14370.

(10) Kim, Y.; Klein, M.; Ren, S.; Chang, Y.; Luo, H.; Samarth, N.; Furdyna, J. Phys. Rev. $B$ 1994, 49, 7262 . 